\title{
MODELAGEM MATEMÁTICA TRANSIENTE E TRIDIMENSIONAL DE SOLDAGEM A ARCO*
}

João Lucas L. Cortez Alves ${ }^{1}$ Marcelo de Aquino Martorano ${ }^{2}$

\section{Resumo}

Foi implementado um modelo matemático transiente e tridimensional para prever os campos de temperatura e composição presentes no sólido e poça de fusão durante um processo de soldagem a arco. O modelo foi desenvolvido com base nas equações de conservação macroscópicas de calor, massa e espécies químicas, que foram solucionadas pelo método dos volumes finitos. Os resultados do modelo mostraram boa aderência com soluções analíticas. Foram apresentados os efeitos de mudança na velocidade e potência de soldagem na distribuição de temperatura obtida e no formato da poça de fusão para soldagem em uma chapa de liga Al$6,5 \% \mathrm{Cu}$.

Palavras-chave: Soldagem a Arco; Modelagem Matemática; Método dos Volumes Finitos

\section{TRANSIENT THREE-DIMENSIONAL MATHEMATICAL MODELING OF ARC WELDING}

\begin{abstract}
A transient and three-dimensional mathematical model was implemented to predict the temperature and composition fields in the solid and liquid phases during an arc welding process. The mathematical model was developed from the macroscopic conservation equations for heat, mass, and chemical species, that were then solved using the finite volume method. The model was validated by comparison with available analytical solutions. The effect of changes in welding speed and energy input in the temperature field and weld pool shape is also presented for the case of bead on plate welding of an $\mathrm{Al}-6,5 \% \mathrm{Cu}$ alloy.
\end{abstract}

Keywords: Arc Welding; Mathematical Modeling; Finite Volume Method.

Mestrando em Eng. Metalúrgica e de Materiais, Universidade de São Paulo, São Paulo, SP.

Professor em Eng. Metalúrgica e de Materiais, Universidade de São Paulo, São Paulo, SP. 


\section{INTRODUÇÃO}

Processos de soldagem com arco elétrico são muito utilizados para junção de metais na indústria de transformação. Esses processos envolvem diversos fenômenos eletromagnéticos e de transporte de calor, espécies químicas, massa e quantidade de movimento. No entanto, a observação e estudo experimental destes fenômenos, especialmente aqueles que ocorrem dentro da poça de fusão, é muito difícil devido à grande quantidade de energia dispensada pelo arco elétrico e à opacidade do metal líquido. Isto dificulta a obtenção, por métodos experimentais, de informações relativas à distribuição de temperatura e composição e ao escoamento no interior da poça de fusão durante a evolução do processo. A modelagem matemática torna-se, então, uma ferramenta importante para o entendimento do que acontece dentro da poça de fusão e dos princípios físicos e forças motrizes que guiam o processo de soldagem.

Diversas variáveis podem afetar a penetração da poça de fusão, a geometria do cordão e a qualidade geral da solda resultante nos processos de soldagem a arco. Dentre elas, podem ser citadas a velocidade de soldagem, a orientação da tocha, a polaridade, o gás de proteção e o aporte térmico, definido como

$$
\frac{\eta V I}{v}
$$

onde $\boldsymbol{V}$ é a tensão, $\boldsymbol{I}$ a corrente, $\square$ a eficiência energética do processo e $\square$ a velocidade de soldagem. Esta grandeza representa o total de energia disponível para a soldagem, é função das variáveis de processo escolhidas e afeta principalmente o ciclo térmico e a penetração da poça de fusão.

Até a década de 1970, investigações sobre a modelagem matemática dos fenômenos de transporte na soldagem se restringiam a soluções analíticas simplificadas, que eram utilizadas para prever as temperaturas em regiões mais distantes da poça de fusão [18]. No início da década de 1980, o avanço computacional permitiu o desenvolvimento de modelos mais complexos e precisos que consideram inclusive a convecção do fluido na poça de fusão. O objetivo do presente trabalho é propor, implementar e aplicar um modelo matemático transiente e tridimensional para prever a transferência de calor e espécies químicas na soldagem a arco. O modelo será construído com base nas equações gerais de conservação macroscópicas desenvolvidas para sistemas multifásicos e permitirá a obtenção dos campos de temperatura, composição do líquido e sólido e fração de sólido durante a soldagem. O modelo será utilizado para verificar o efeito da velocidade de soldagem da potência do arco e do aporte térmico na soldagem de uma liga do sistema Al-Cu.

\section{MATERIAIS E MÉTODOS}

A partir de equações de conservação macroscópica de energia, massa e espécies químicas, foi desenvolvido um modelo matemático transiente e tridimensional para o processo de soldagem a arco de uma chapa. Neste modelo, foram considerados a fusão do sólido da chapa e a sua solidificação posterior, a fusão e solidificação do eutético, o transporte de calor por condução no sólido e líquido e a difusão de soluto no interior do líquido. O aporte de energia foi aproximado pela fonte de distribuição gaussiana de calor proposta por Eagar e Tsai (2). As seguintes hipóteses simplificadoras foram usadas para a obtenção da forma diferencial final das equações do modelo: 
- O sistema consiste de uma liga binária eutética onde podem existir três fases ou microconstituintes: líquido $(\boldsymbol{I})$, sólido primário $(\boldsymbol{s})$, eutético $(\boldsymbol{e})$.

- Calor específico, condutividade térmica e densidade são constantes e iguais para todas as fases presentes.

- Há equilíbrio térmico local entre sólido e líquido.

- Há equilíbrio termodinâmico local na interface sólido/líquido.

- Convecção desprezível no líquido.

- Difusão macroscópica de soluto desprezível no líquido e sólido.

- Difusão microscópica de soluto desprezível no sólido, mas considerada no líquido.

- O sólido eutético (e) tem composição constante.

O desenvolvimento do modelo matemático inicia-se pelas formas gerais das equações de conservação macroscópicas desenvolvidas pelo método das médias volumétricas e aplicadas à solidificação por $\mathrm{Ni}$ e Beckermann [11]. O uso deste método permite tratar todo o sistema envolvendo região sólida, zona pastosa e região líquida com o mesmo conjunto de equações (método de domínio único). Deste modo, não é necessário conhecer a priori a localização e formato da interface sólido/líquido. As equações de conservação gerais foram aplicadas a cada um dos três constituintes considerados e simplificadas de acordo com as hipóteses descritas anteriormente, resultando em :

$$
\begin{array}{r}
\rho c \frac{\partial T}{\partial t}=K \nabla^{2} T-\rho L_{f} \frac{\partial \epsilon_{l}}{\partial t} \\
\rho \frac{\partial \epsilon_{s}}{\partial t}=\Gamma_{s} \\
\rho \frac{\partial \epsilon_{e}}{\partial t}=\Gamma_{e} \\
\rho \frac{\partial \epsilon_{l}}{\partial t}=-\Gamma_{t} \\
\frac{\partial}{\partial t}\left(\epsilon_{s}\left\langle C_{s}\right\rangle^{s}\right)=k_{p}\left\langle C_{l}\right\rangle \frac{\partial \epsilon_{s}}{\partial t} \\
\frac{\partial}{\partial t}\left(\epsilon_{l}\left\langle C_{l}\right\rangle^{l}\right)=-k_{p}\left\langle C_{l}\right\rangle \frac{\partial \epsilon_{s}}{\partial t}-C_{e} \frac{\partial \epsilon_{e}}{\partial t} \\
T=T_{f}+m_{l}\left\langle C_{l}\right\rangle^{l} \\
\Gamma_{t}=\Gamma_{e}+\Gamma_{s}
\end{array}
$$

Onde $\boldsymbol{T}$ é a temperatura no volume elementar, $\boldsymbol{c}$ é a capacidade térmica, $\square \mathrm{a}$ densidade, $\boldsymbol{L}_{\boldsymbol{f}} \mathrm{O}$ calor latente de fusão, $\boldsymbol{T}_{\boldsymbol{f}}$ a temperatura de fusão do metal puro, $\boldsymbol{m}_{\boldsymbol{l}}$ a inclinação da linha liquidus $e\left\langle C_{k}\right\rangle{ }^{k}$ o teor de soluto em uma fase $\boldsymbol{k}$, sendo que os símbolos $\left\langle\mathbf{X}_{\mathbf{k}}\right\rangle^{\mathbf{k}}$ indicam a média volumétrica intrínseca de uma quantidade $X$ na fase $\boldsymbol{k}$.

Foi considerado o processo de soldagem a arco autógeno de um cordão de solda linear sobre uma chapa de espessura considerada média segundo a definição de Rosenthal [1], em posição plana (Figura 1). No tempo $t=0$, a tocha de soldagem parte de uma extremidade da chapa, na posição da metade de sua largura, percorrendo a superfície desta chapa ao longo de seu comprimento. A chapa está 
inicialmente à temperatura ambiente $\boldsymbol{T}_{a r}=25^{\circ} \mathrm{C}$ e sua superfície troca constantemente calor com o ambiente de acordo com um coeficiente de transporte de calor $\boldsymbol{h}=100 \mathrm{~W} / \mathrm{m}^{2}{ }^{\circ} \mathrm{C}$, calculado para o caso da convecção natural. $\mathrm{O}$ aporte de calor gerado pelo arco elétrico foi modelado pela distribuição gaussiana proposta por Eagar e Tsai [2], utilizada como condição de contorno para a equação da conservação de energia (Eq (2)).

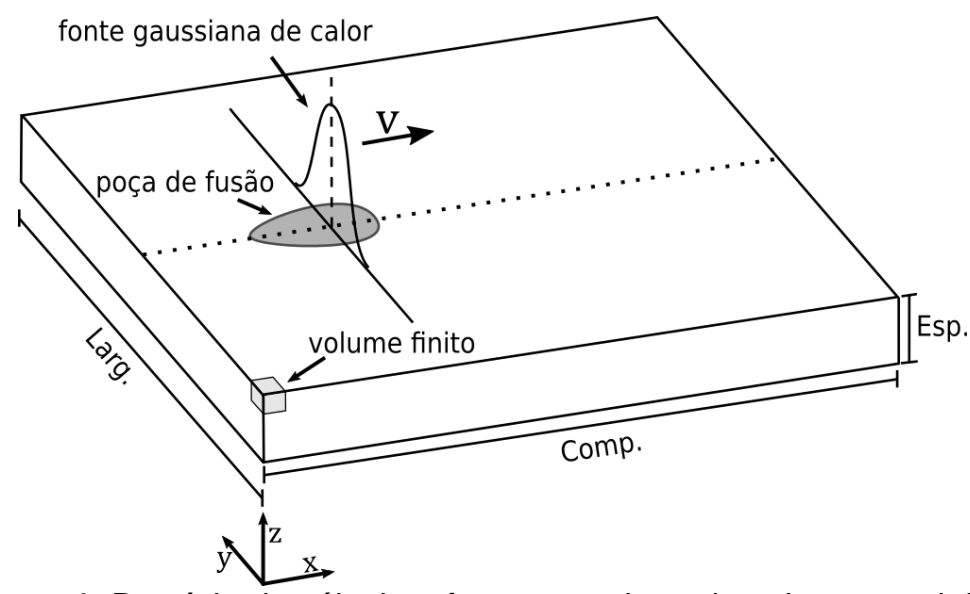

Figura 1. Domínio de cálculo e fonte gaussiana de calor na posição da tocha.

O sistema de equações apresentado foi solucionado numericamente em um domínio tridimensional pelo método dos volumes finitos, na sua formulação implícita. O domínio de cálculo, que consiste de uma placa, foi discretizado por uma malha de $100 \times 65 \times 15$ volumes cúbicos uniformes contendo um nó em seu centro. O conjunto de equações algébricas discretizadas foi solucionado pelo método iterativo de Gauss-Seidel. O código computacional para a resolução numérica das equações foi escrito em linguagem $\mathrm{C}++$, utilizando múltiplas linhas de execução ("multithreading") para paralelização dos cálculos. No código computacional foi também construído uma saída gráfica especial para visualização de parte dos resultados. As simulações foram conduzidas em um computador pessoal com processador Intel Core i7 e 16GB de memória RAM e o tempo médio de cada simulação foi de 10 min para um passo de tempo de 0.005 s..

\section{RESULTADOS E DISCUSSÃO}

\subsection{Simulação da Soldagem a Arco de uma Liga do Sistema Al-Cu}

As simulações foram realizadas em uma liga Al-6,5\%Cu (ASTM 2219) que faz parte de um sistema binário simples com um patamar eutético e um diagrama de fases bem documentado, além de ser comum na prática industrial da soldagem de peças em alumínio pelos processos de soldagem a arco. As propriedades desta liga estão apresentadas na Tabela 1.

Alguns parâmetros de processo apropriados para a soldagem da liga Al$6.5 \% \mathrm{Cu}$, definidos nos experimentos de Kou e Yang [14], foram escolhidos para as simulações do presente trabalho e estão apresentados na Tabela 2.

Tabela 1. Propriedades da liga escolhida

\begin{tabular}{lccc}
\hline Propriedades liga Al6.5\%Cu & Símbolo & Valor & Unidade \\
\hline Densidade & 0 & 27050 & $\mathrm{Kg} / \mathrm{m}^{3}$ \\
\hline Condutividade térmica & $\boldsymbol{k}$ & 170 & $\mathrm{~W} / \mathrm{m}^{\circ} \mathrm{C}$ \\
\hline
\end{tabular}




\begin{tabular}{llcc}
\hline Calor específico & $\boldsymbol{c}$ & 864 & $\mathrm{~J} / \mathrm{Kg}{ }^{\circ} \mathrm{C}$ \\
\hline Calor latente de fusão & $\boldsymbol{L}_{f}$ & 425 & $\mathrm{KJ}$ \\
\hline
\end{tabular}

Fonte: ASM Internacional 1990, Holt 1996.

Tabela 2. Parâmetros e condições impostas

\begin{tabular}{lccc}
\hline Condições do ambiente & Símbolo & Valor & Unidade \\
\hline Temperatura ambiente & $\boldsymbol{T}_{\boldsymbol{a r}}$ & 25 & ${ }^{\circ} \mathrm{C}$ \\
\hline Coeficiente de transferência de calor & $\boldsymbol{h}$ & 100 & $\mathrm{~W} / \mathrm{m}^{2}{ }^{\circ} \mathrm{C}$ \\
\hline Parâmetros do Processo & & & \\
\hline Eficiência na transferência de calor & $\boldsymbol{\eta}$ & $80 \%$ & \\
\hline Tensão & $\boldsymbol{V}$ & 28 & Volts \\
\hline Corrente & $\boldsymbol{I}$ & 285 & $\mathrm{Amps}$ \\
\hline Coeficiente de distribuição de calor & $\square$ & 3.5 & $\mathrm{~mm}$ \\
\hline Velocidade de avanço da tocha & $\square$ & 7 & $\mathrm{~mm} / \mathrm{s}$ \\
\hline Dimensões da placa & \multicolumn{3}{c}{$100 \times 65 \times 15 \mathrm{~mm}$} \\
\hline
\end{tabular}

A Figuras 2(a), (b) e (c) mostram os campos de temperatura, fração de líquido, e composição da fase líquida, respectivamente, na superfície superior da chapa e em uma secção longitudinal após 7 segundos. A posição da fonte de calor é denotada pela seta vermelha.
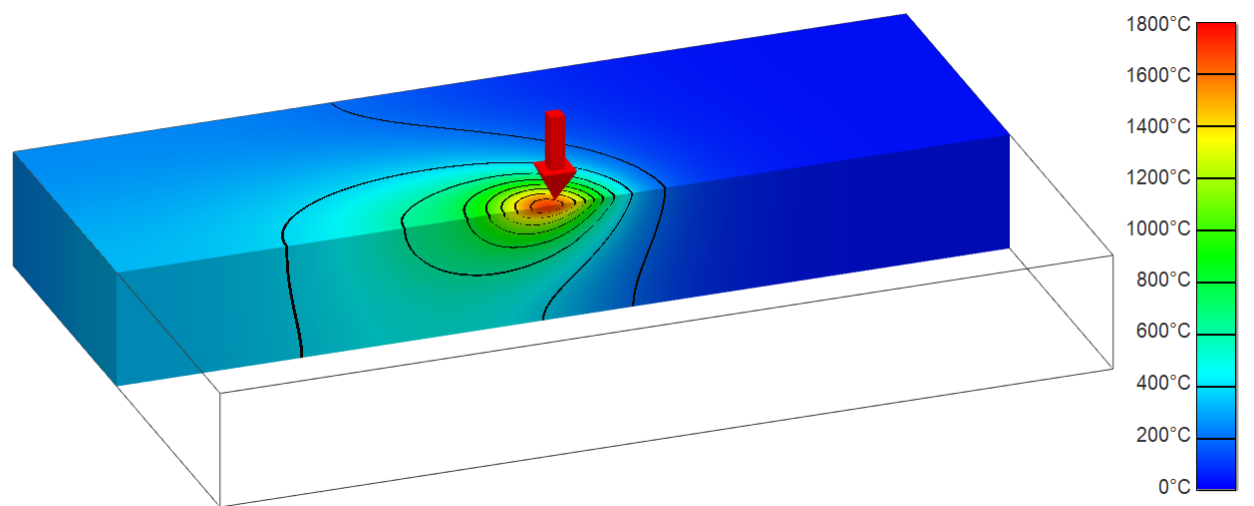

(a)
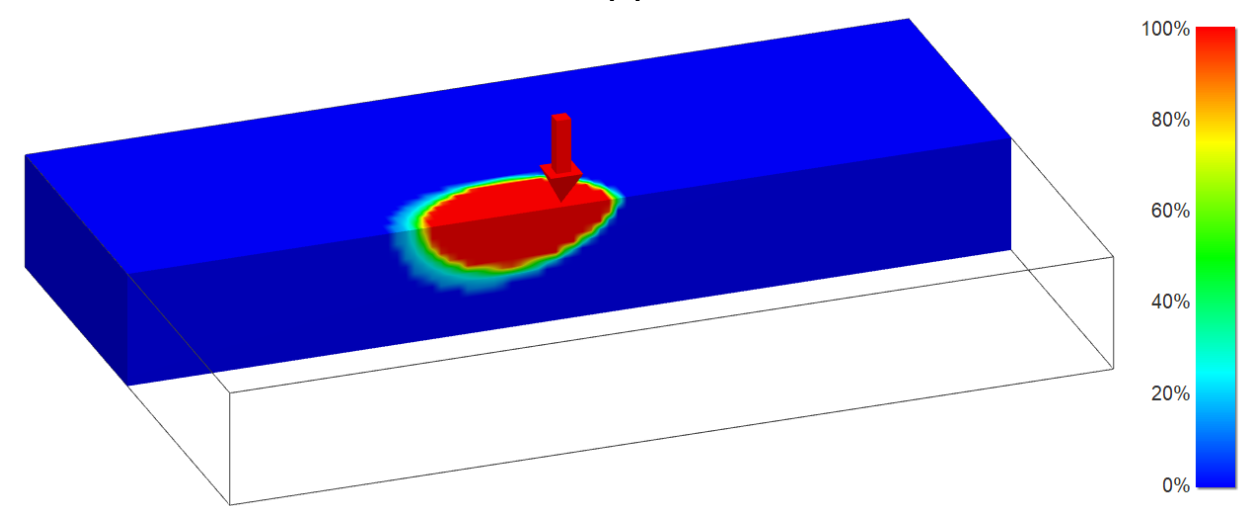

(b) 

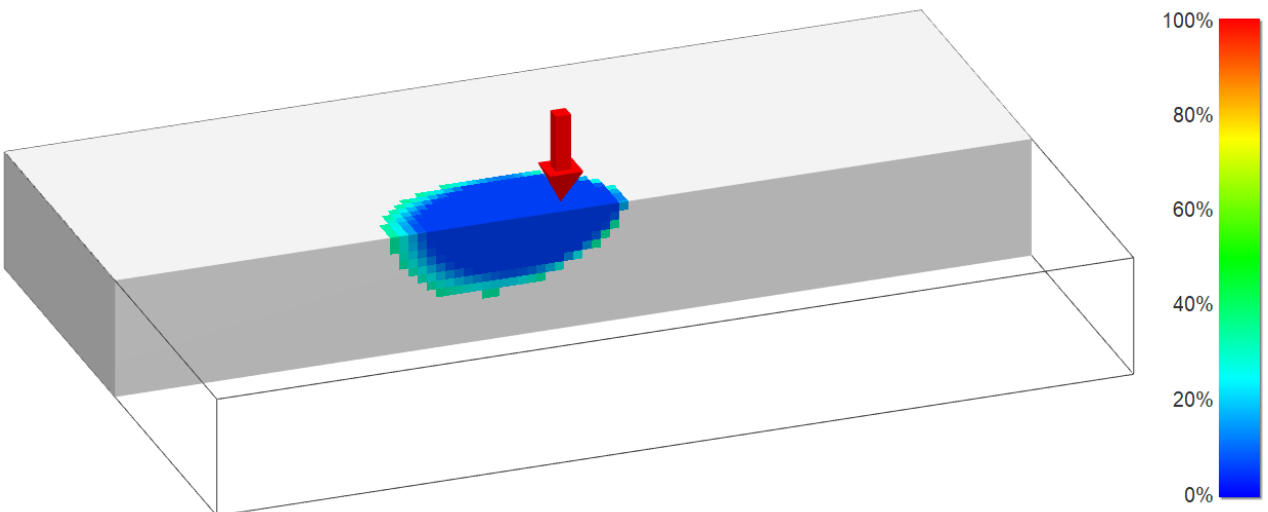

(c)

Figura 2. Resultados do modelo para simulação da soldagem de uma placa de Al-6,5\%Cu: (a) temperatura; (b) fração de líquido e (c) \%Cu na fase líquida ( $t=7 \mathrm{~s}$ ).

A figura 3 mostra o perfil de temperaturas ao longo da linha central da chapa em diferentes instantes de tempo. Os perfis tendem para um mesmo formato indicando que o processo está tendendo a um estado quasi-estacionário, o que permite a comparação com soluções analíticas clássicas, como a de Rosenthal [1], para o transporte de calor durante a soldagem.

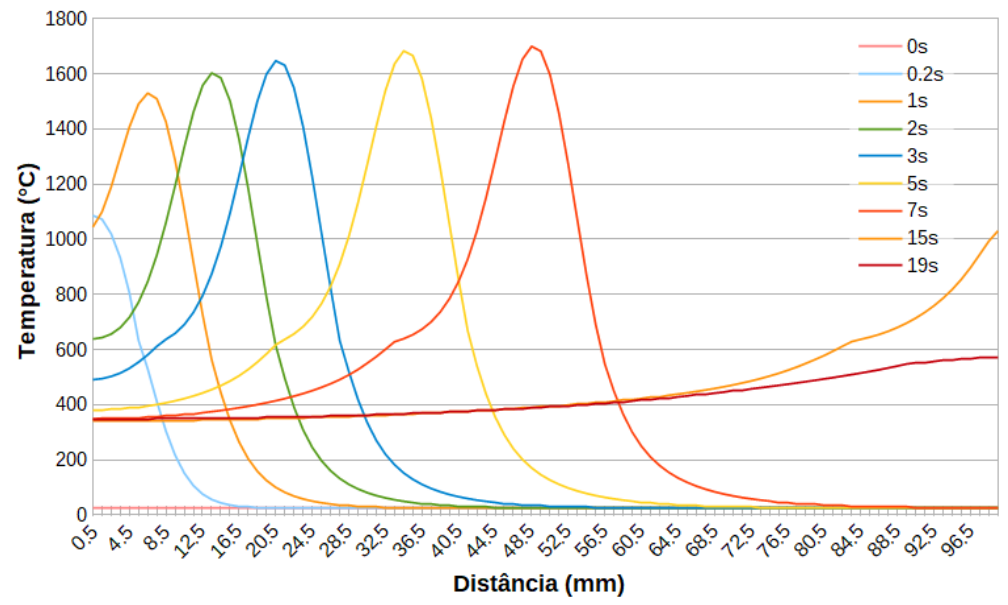

Figura 3. Temperatura na linha central em diversos tempos.

Para uma melhor comparação entre os resultados do modelo desenvolvido e da solução analítica clássica, uma nova simulação foi realizada com valores praticamente nulos para os parâmetros: $L_{f}(0.01 \mathrm{~J})$ desprezando o efeito do calor latente, $\boldsymbol{h}\left(0.01 \mathrm{~W} / \mathrm{m}^{2} \mathrm{C}\right)$ desprezando o efeito da perda de calor para o ambiente e $\square$ $(0.001 \mathrm{~mm})$ simulando uma fonte de calor puntiforme. O modelo modificado desta forma incorpora as hipóteses das soluções de Rosenthal [1]. A Figura 7 mostra uma comparação dos resultados obtidos pelo modelo original e sua versão modificada com as soluções analíticas para chapa grossa (sólido semi-infinito) e chapa de espessura média para a temperatura na linha central. Como estas soluções analíticas baseiam-se em fontes de calor puntiformes para representar a tocha de soldagem, a temperatura tende ao infinito quando a distância da fonte de calor se aproxima da posição da tocha [6]. A solução para chapa de espessura média apresenta uma boa concordância com os resultados do modelo modificado. 

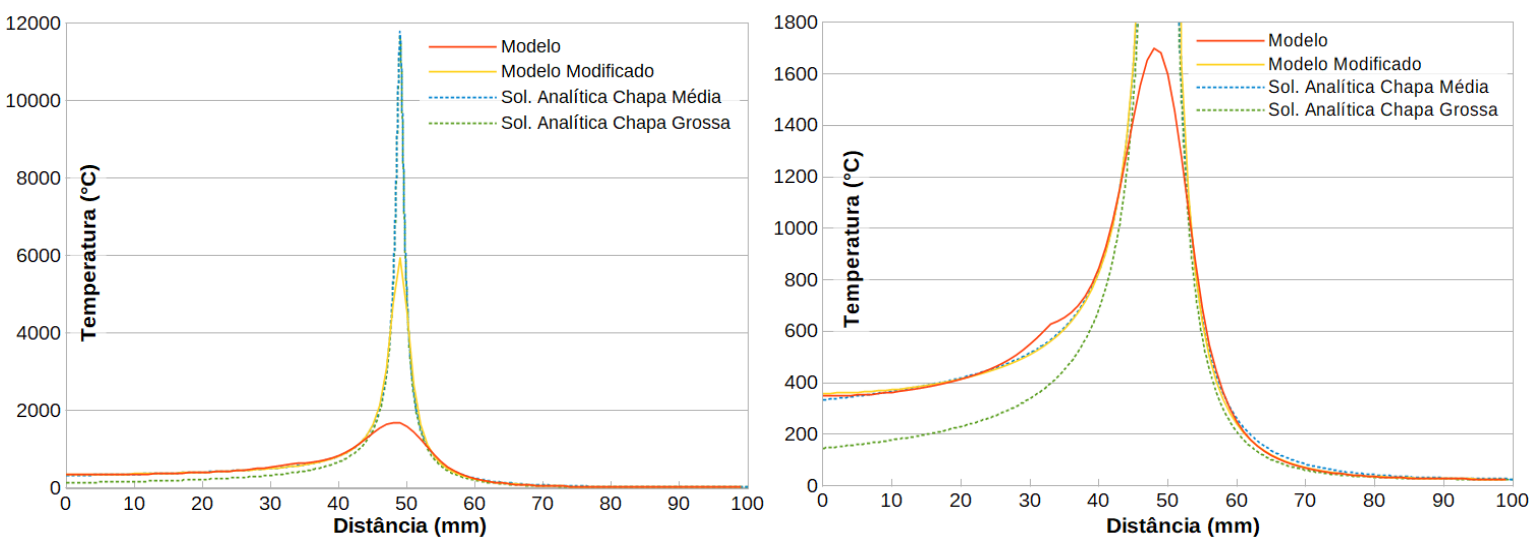

Figura 7. Comparação entre temperaturas previstas pelo modelo e soluções analíticas de Rosenthal em duas escalas diferentes de temperatura.

Os gráficos da figura 8 apresentam perfis de temperatura, fração volumétrica de sólido primário, sólido eutético e líquido ao longo da linha central da placa no instante $t=7 \mathrm{~s}$. O pequeno pico observado nas curvas de temperatura próximo a $600^{\circ} \mathrm{C}$ é causado pela liberação de calor latente durante a solidificação primária e eutética.

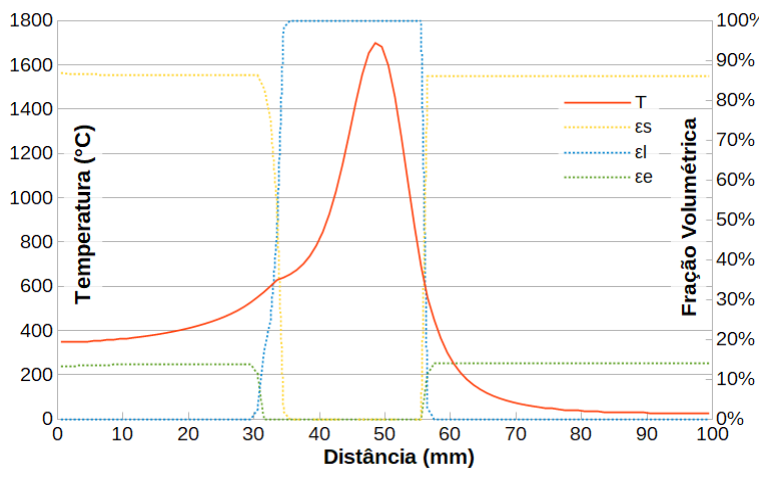

(a)

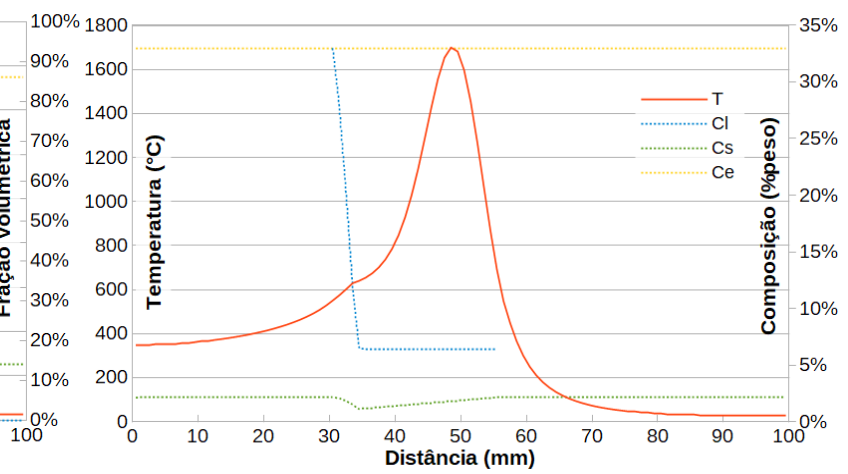

(b)

Figura 8. Perfis calculados pelo modelo proposto ao longo da linha central da chapa no instante $t=$ 7s: (a) temperatura e fração de sólido primário, sólido eutético e fração de líquido; (b) temperatura e concentração de Cu no líquido, no sólido primário e no sólido eutético.

\subsection{Efeitos da Velocidade de Soldagem, Potência e Aporte Térmico}

Para se estudar os efeitos da alteração dos parâmetros de processo novas simulações foram realizadas. Três casos adicionais foram considerados, um com o dobro de velocidade de soldagem $\boldsymbol{v}$ (figuras 9 e 10), outro com o dobro de potência de soldagem $V \times I$ (figuras 11 e 12) e, finalmente, um onde foram realizadas as duas alterações simultâneamente (figuras 13 e 14), assim mantendo constante o aporte térmico (Eq. 1). As figuras 9, 11 e 13 mostram uma comparação entre os campos de temperatura obtidos com os resultados para os parâmetros iniciais na parte superior e nos novos casos na parte inferior. De forma similar as figuras 10, 12 e 14 comparam o formato das poças de fusão pela fração de fase líquida obtida. 


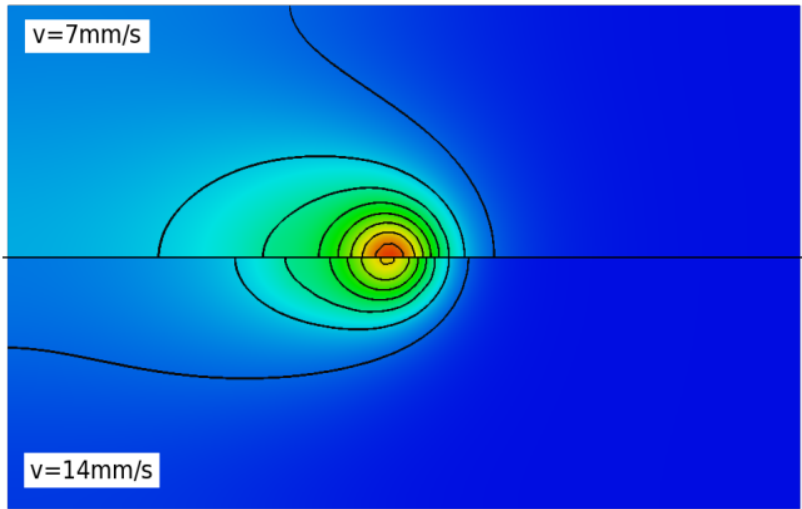

(a)

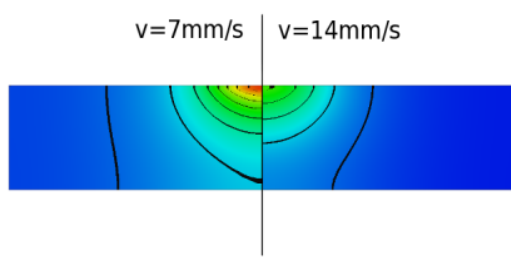

(b)

Figura 9. Temperatura na (a) superfície da chapa e (b) na seção transversal para duas velocidades de soldagem diferentes.

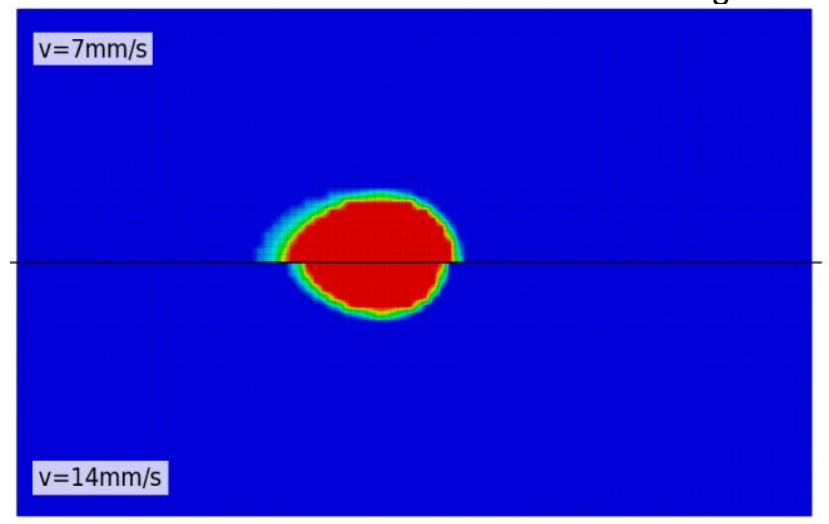

(a)

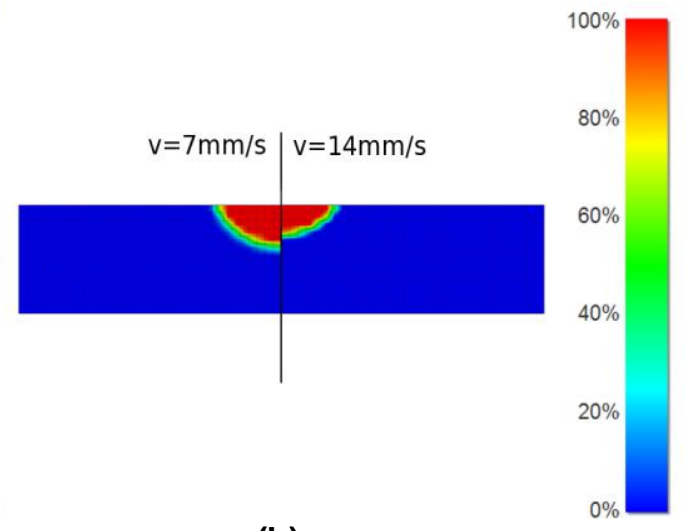

Figura 10. Fração de fase líquida na (a) superfície da chapa e (b) na seção transversal para duas velocidades de soldagem diferentes.

Com o aumento de velocidade nota-se que a poça de soldagem diminui (figura 10) bem como a extensão da zona aquecida (figura 9), como esperado devido ao menor aporte térmico (eq.1). Como a velocidade foi dobrada em relação ao caso original as figuras mostram os processos em tempos diferentes (7s e $3.5 \mathrm{~s}$ ) respectivamente.

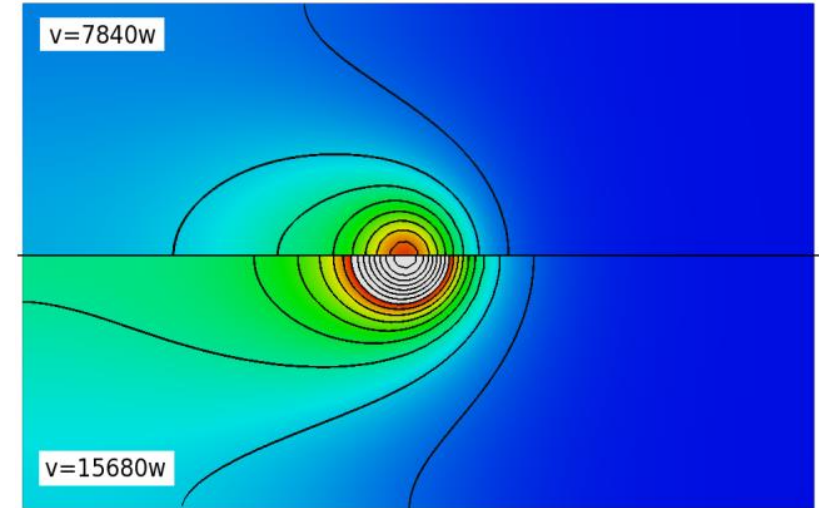

(a)

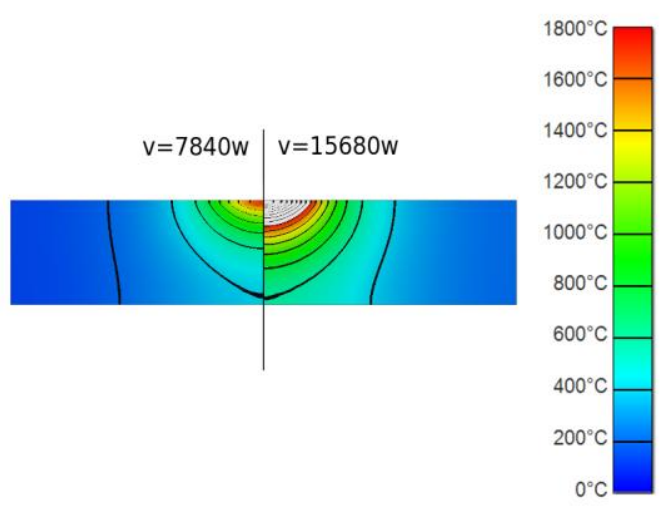

(b)

Figura 11 Temperatura na superfície com aumento da potência na (a) superfície da chapa e (b) na seção transversal. 


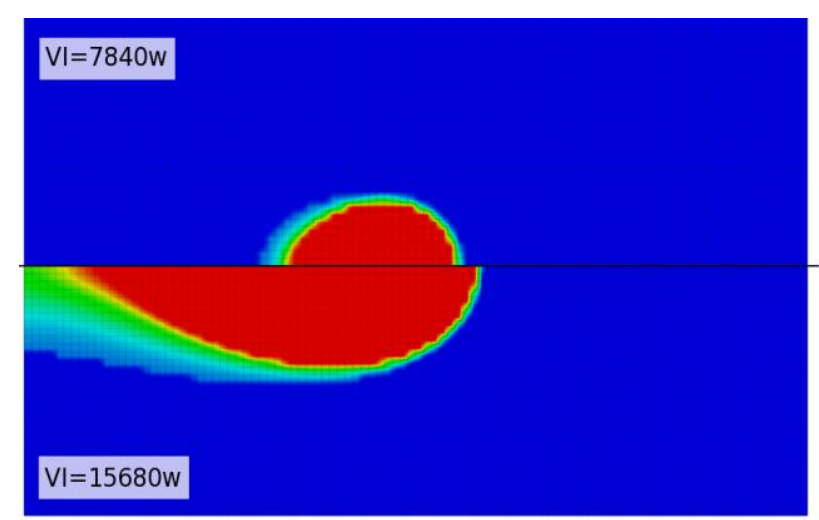

(a)

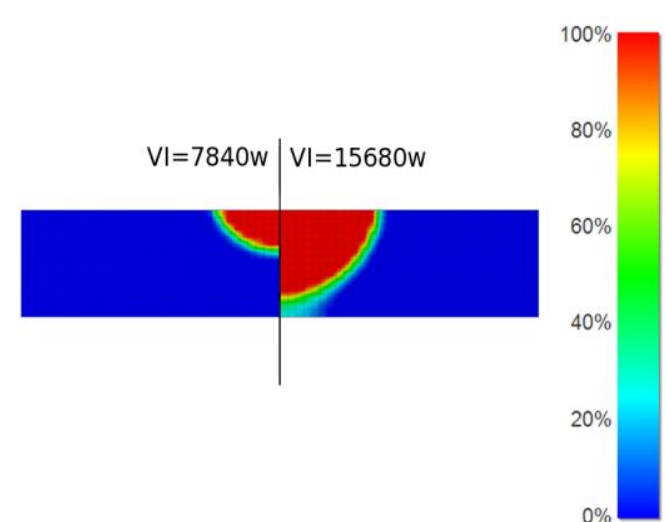

(b)

Figura 12. Fração de fase líquida com aumento da potência na (a) superfície da chapa e (b) na seção transversal.

Com o aumento da potência da fonte gaussiana de calor a poça de soldagem aumenta e assume um formato de gota (figura 12a), com os contornos da parte posterior se aproximando de linhas retas. A largura e penetração da poça de fusão, bem como o tamanho da zona aquecida e o pico de temperatura (figura 11) aumentaram de forma expressiva.

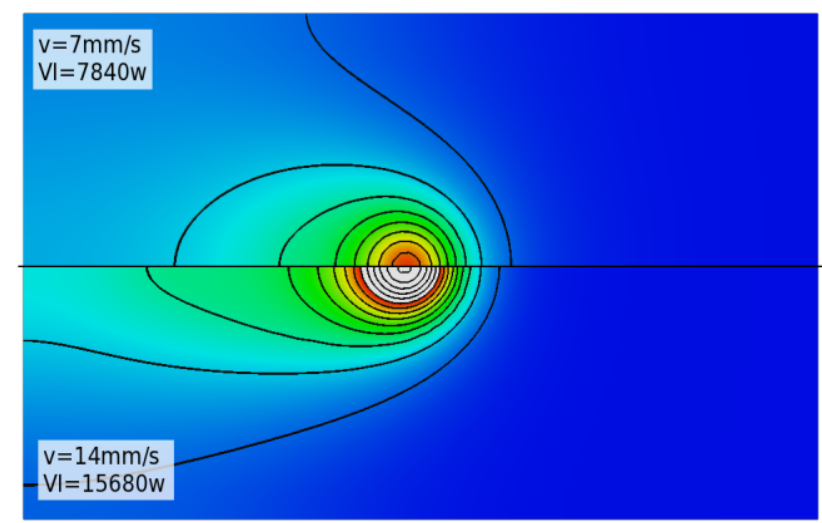

(a)

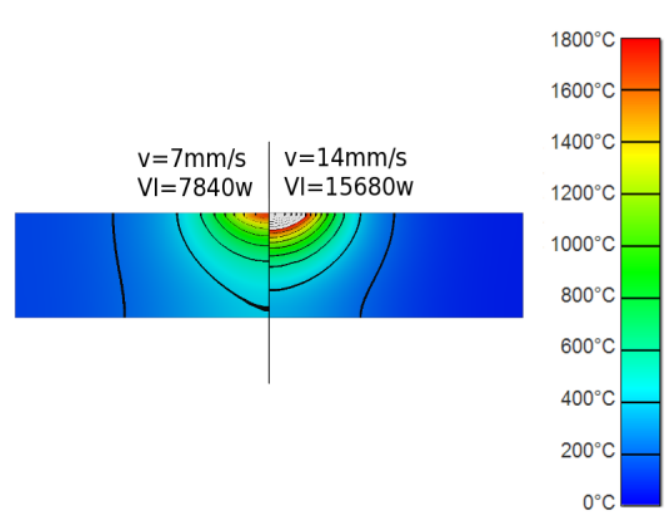

(b)

Figura 13. Temperatura na superfície com aumento da velocidade e potência na (a) superfície da chapa e (b) na seção transversal.

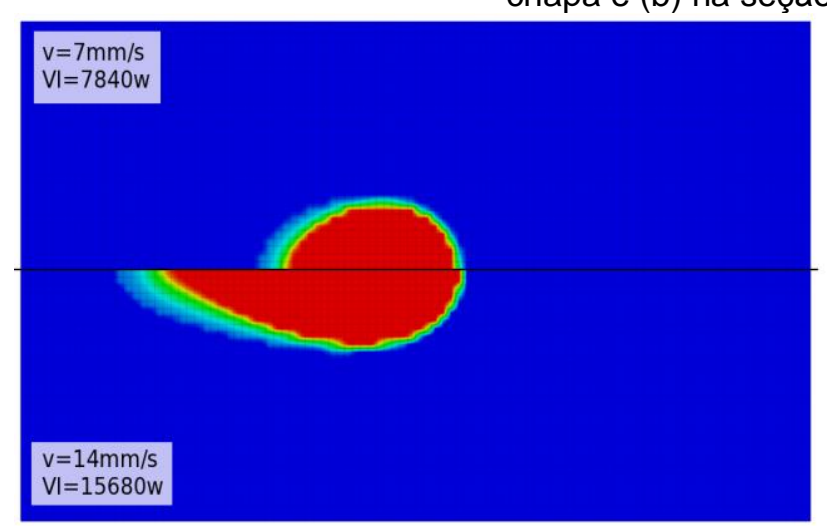

(a)

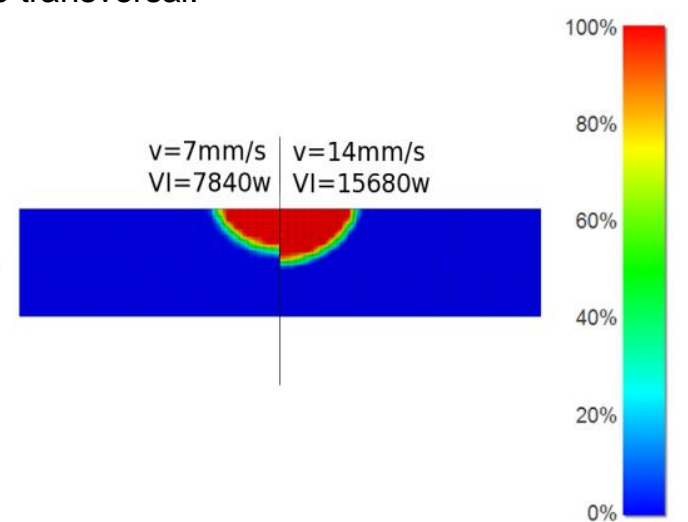

(b)

Figura 14. Fração de fase líquida com aumento da velocidade e potência na (a) superfície da chapa e (b) na seção transversal..

Com o aumento simultâneo da potência da fonte de calor e da velocidade de soldagem a poça de soldagem se alonga, se tornando menos elíptica e mais com 
formato de gota (figura 14a) mas mantém uma largura similar. A temperatura no centro da poça de fusão no entanto é bem superior ao caso inicial (figura 13).

A figura 15 apresenta uma comparação dos ciclos térmicos obtidos nos três casos novos e o modelo com os parâmetros iniciais, para um ponto no centro da placa. $O$ instante 0 representa 0 momento em que a tocha de soldagem está diretamente acima do ponto.

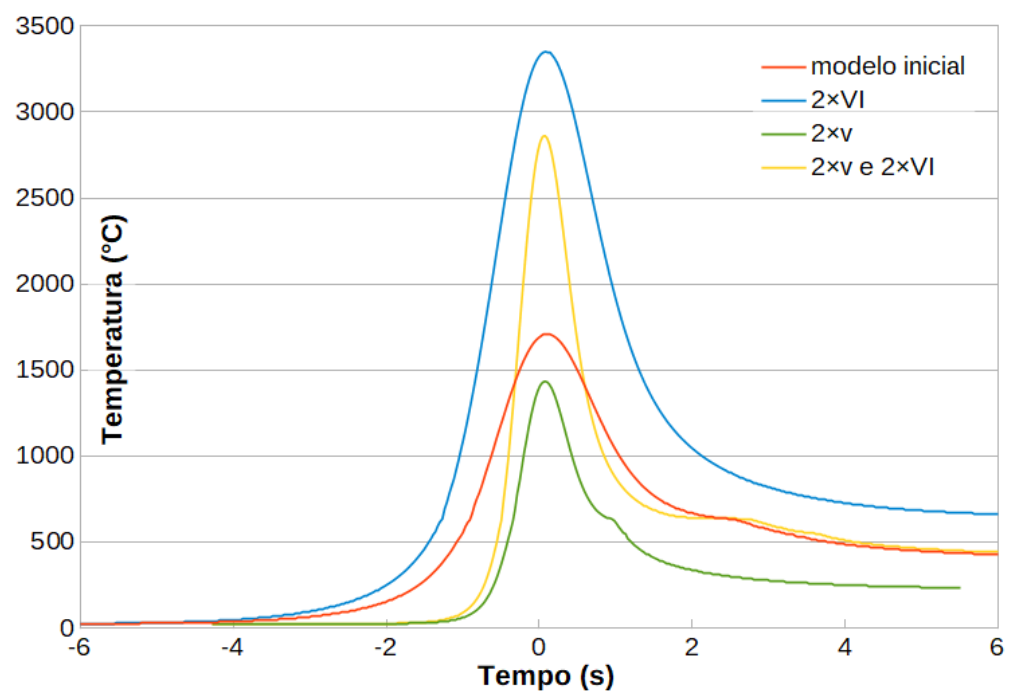

Figura 15. Comparação entre os ciclos térmicos para os 4 casos.

O caso com o dobro da potência $(2 \times V I)$ apresenta as maiores temperaturas e o caso com a maior velocidade $(2 \times v)$ as menores, assim como esperado, dada a variação de aporte térmico. Já o caso com o dobro de velocidade e potência apresenta um pico maior (em relação ao modelo original) porém, mais estreito mantendo a área abaixo da curva muito próxima ao caso inicial. A temperatura neste caso também é muito próxima da obtida nas condições iniciais, logo após alguns segundos. Isso se deve, é claro, ao fato da mesma quantidade total de energia estar sendo depositada nos volumes nestes dois casos.

\section{CONCLUSÃO}

Uma versão simplificada do modelo desenvolvido mostrou boa aderência com a solução analítica de Rosenthal [1]. Já a versão completa, que leva em consideração os efeitos do calor latente, redistribuição de soluto, perda de calor para o ambiente, e utiliza uma distribuição de calor mais próxima do real, se mostrou útil para os estudo do efeito da variação da velocidade de soldagem e potência da fonte de calor, mostrando a variação da largura e penetração da poça de soldagem. A comparação entre 4 casos diferentes também mostrou a alteração no formato da poça de fusão, passando de um formato elíptico para a forma de gota com o aumento da potência. A mudança na extensão da zona aquecida e nos valores dos picos de temperatura também foi mostrada.

\section{REFERÊNCIAS}

1 ROSENTHAL, D. The theory of moving sources of heat and its application to metal treatments. ASME. [S.I.], 1946.

2 EAGAR TW, TSAI NS. Temperature fields produced by traveling distributed heat 
sources. Welding journal. 1983 Dez 1;62(12):346-55.

3 BRANDI SÉRGIO D; WAINER, Emílio; Mello Fábio D H. Soldagem: processos e metalurgia. Edgar Blucher, São Paulo, 1992.

4 SCHEIL, E. Nonequilibrium solidification. Z. fetallk, v. 34, p. 70-74, 1942.

5 SUNDERLAND, JE; SH Cho. Heat-conduction problems with melting or freezing. J. Heat Transfer, v. 91, p. 421-426, 1969.

6 GRONG, Oystein. Metallurgical modelling of welding. Institute of Materials, London, UK, 1997.

7 WELDING Handbook. Volume 2: Welding Processes, Part 1. [S.I.]: American Welding Society, 2004. ISBN 0871717298.

8 LIENERT, Thomas J. Welding Fundamentals and Processes (Asm Handbook, Volume 6A). [S.I.]: ASM International, 2011. ISBN 1615031332.

9 LIPPOLD, John C. Welding metallurgy and weldability. [S.I.]: John Wiley \& Sons, 2014.

10 PATANKAR, Suhas. Numerical heat transfer and fluid flow. [S.I.]: CRC press, 1980.

11 NI JUN; BECKERMANN, Christoph. A volume-averaged two-phase model for transport phenomena during solidification. Metallurgical Transactions B, Springer, v. 22, n. 3, p. 349-361, 1991.

12 WANG CY; BECKERMANN, C. A multiphase solute diffusion model for dendritic alloy solidification. Metallurgical Transactions A, The Minerals, Metals \& Materials Society, v. 24, p. 2787-2787, 1993.

13 KURZ, W.; FISHER, D.J. Fundamentals of solidification. [S.I.]: Trans Tech Publications, 1998. ISBN 9780878498048.

14 YANG YK; KOU, S. Fusion-boundary macrosegregation in dissimilar-filler metal al-cu welds. Welding Journal, v. 86, n. 11, p. 331, 2007.

15 USHIO M; WU, CS. Mathematical modeling of three-dimensional heat and fluid flow in a moving gas metal arc weld pool. Metallurgical and materials transactions B, Springer, v. 28,n. 3, p. 509-516, 1997.

16 OREPER GM; SZEKELY, J. Heat-and fluid-flow phenomena in weld pools. Journal of Fluid Mechanics, Cambridge University Press, v. 147, p. 53-79, 1984.

17 ZACHARIA T; DAVID, SA; Vitek JM; DebRoy T. Weld pool development during gta and laser beam welding of type 304 stainless steel; part i-theoretical analysis. Welding Journal,v. 68, n. 12, 1989.

18 JONSSON PG; SZEKELY, J; Choo RTC; Quinn TP. Mathematical models of transport phenomena associated with arc-welding processes: a survey. Modelling and Simulation in Materials Science and Engineering, IOP Publishing, v. 2, n. 5, p. 995, 1994. 